\title{
MozEx: Web? Arts? Tech? Experience?
}

\author{
Luca M. Damiani \\ Tate / London College of Communication \\ Tate / University of the Arts London, UK \\ luca.damiani@tate.org.uk \\ I.damiani@/cc.arts.ac.uk
}

\author{
Irini Papadimitriou \\ Victoria \& Albert Museum \\ V\&A London, UK \\ i.papadimitriou@vam.ac.uk
}

\begin{abstract}
We constantly see art and technology intertwined in digital art, net-art, creative coding and current dialogues within visual culture. Fully opening to this relationship, MozEx is an art exhibition with a 21st-century twist. Curated by the digital learning teams at both the Tate and the V\&A in 2016 (in collaboration with the Mozilla Foundation and Mozilla Festival), it included fifty-four dynamic digital artworks that spanned many disciplines and media. In this paper we look at reviewing our co-working approach as MozEx producers and curators, looking at the constructive cross-exchange of art, web and tech that was created and built-up during this project. The paper opens a critical angle on what it means to form a contemporary and collaborative exhibit project, what critical questions are asked and how the cross-connection with art, tech, culture and web can be key to open-up more constructive gallery and also non-gallery setting, in a physical space and/or on a digital platform.
\end{abstract}

Art. Technology. Web. Collaboration. Experience. Exhibition. Exchange.

\section{INTRODUCTION}

Art and technology are merging more and more, and the open web-community is growing at various levels and directions of cross-disciplinary collaborations and open-participation (Mihailidis 2014) and also different way of seeing and visioning curation (Graham \& Cook 2010, Krysa 2006).

MozEx (Ex stands for exhibition, experience and exchange) considers the interconnection between artistic practice, technological methodologies and the web-community. It is about art, as it is about technology, as it is about people.

The exhibit explores links between art, society, and the digital world. Created by both individual practitioners and cross-disciplinary collaborations, MozEx navigates the value of art to society. This is done through a combination of diverse artistic practices and approaches, sharing different voices about society and the web, as well as considering and reviewing the concept and politics of the openweb (Soep 2014).

Fifty-four artworks were selected around five key issues that related to the thematic of the Mozilla Festival 2016: web literacy, digital inclusion, open innovation, decentralisation, online privacy and security. These ingredients were already part of digital art arguments and questions that digital learning teams at both Tate and V\&A were exploring in their practice-based digital-art programmes. Both teams review the relationship that artistic practice and technology have, and what conversations shape within the cross-disciplinary practice and intervention.

MozEx reflected and emphasised collaboration, open-source practices and the critical thinking around the exploration of new technological worlds (Greengard 2015), big data (Stackowiak et al. 2015) post-humanism approaches (Najar 2003) and public understanding of the topics. The aim of this project was also to investigate and critically explore the impact of technology in society and culture, with the objective to stimulate open discussions between practitioners and audiences. In this paper we consider the open approach that was taken as part of MozEx and we will share the build-up of its open-philosophy.

\section{CROSS-EXCHANGING}

Digital media and digital artistic practice is an increasing part of our lives. The exchanging of 
digital knowledge and practice with Mozilla was already part of the digital learning approach at Tate and V\&A. We both have been involved with Mozilla and with MozFest before, running digital art workshops and setting-up open-talks about the topic of digital-art technology. We have also participated in Mozilla's Open loT Studio research events, looking at the internet of things and reflecting critically on how art can share the various doubts and arguments around it. However, this was always done as separate entities and focusing on project developed respectively or at Tate or at V\&A. So, MozEx was the first time that we collaborated closely together as MozFest co-designers, as well as thinking how to cross-connect our professional approaches on digital learning.

During the first phase of planning, with MozEx we aimed to structure an engaging and collaborative work which demystified technology and opened up discussions and exchanges. Digital learning within Tate and V\&A have similar aims and similar kinds of practice; MozEx was a great opportunity to connect and work together, questioning commongrounds and shared research interests too. The collaboration with Mozilla opened this possibility, perhaps putting down barriers of institutional competitiveness, and allowing more collaboration and exchange within Tate and V\&A.

Part of the work we both do is about cohesion between art and technology: more bridges you can build and more conversations you can start. This was then an anchored key-point of socio-political identity that we wanted to underline with MozEx. The need for discussion, for sharing voices and views, for forming a healthy debate. As part of the Tate Exchange context and philosophy of open and shared experiences, art and knowledge, MozEx was also a platform to exchange digital art practices to new audiences beyond the art gallery context.

Also, using art-gallery collections as a starting point for the investigation of how digital technology informs contemporary art and design, the digital learning identity develops through making and technological engagement that empowers people to become creators and not just consumers. The nature of digital participation (Biggs 2012) and digital citizenship (Bearden 2016) is changing and shaping in different ways in our society, and the flow between digital producers and digital consumers is also changing. Within MozEx we looked at that area of investigation too, also examining what the affordances of digital technology are for learning through art and artists, and how these can impact digital making.

\section{THE APPROACH}

The open-access, open-source and open-data approach within Mozilla creates a dialogue on the web that cross-over topics, methods and philosophies also within digital art. And so "the open" became the key-principle ingredient of our MozEx approach.

All the work part of MozEx was set on various different Creative Commons (CC) licences (https://creativecommons.org). This opened interesting conversations with the artists and created problems since the outset of the work itself. CC-licenses enable the free distribution of an otherwise copyrighted work (Schuber \& McClean 2002); they grant 'baseline rights', such as the right to distribute the work worldwide for non-commercial (or also commercial depending from the license) purposes, and with or without edits and modification. Artists choose a license according to the conditions they want to be applied to their artwork, so it changes depending from situation to situation. The details of each of these licenses depend on the version and are made up of a selection of different conditions such SA (ShareAlike), NC (Non-commercial) and ND (No Derivates). Many artists interested in MozEx did not submit their work because they wanted their work to be fully copyrighted. This shaped interesting and critical discussions also with Mozilla, and challenged the limits to what "open" might mean in different scenarios and for different people. This primary difficulty inspired the exhibition even more. We reviewed the importance of open culture philosophy and how that was fundamental to be inserted in contemporary artistic practice on and about the web, within the digital art and techdomain.

Each of the fifty-four components of the installation agreed to a 'CC BY-NC' (non-commercial) license and in their work they examined issues facing a free and open internet. The selection was done through an open call for people to become involved which led to a great diversity of submissions from plenty of artists who hadn't been linked to Mozilla or Tate or V\&A before. The open-call was followed by an open-selection on GitHub (https://github.com) with open-feedback, open-communication and open-data. This was reflected in the exhibition itself.

\section{CURATION AND PRODUCTION}

MozEx needed to reflect the diversity of the web and needed to be open to various methodologies in order to engage a wider spectrum of voices and critical perspectives. We wanted to attract artists as well as coders, opening some arguments of the 
identity of contemporary artists working with arts and technology. We got academics, creative technologists and artists that in their work are interested in debating art\&technology relation, care about learning and interactive participation.

It was a challenge to be able to combine so many different perspectives together in one place, with one project, with one call. But the curatorial choice created a very dynamic collection of artworks: writing, photography, video, audio, mix-media, bots. All these extensive methods are the real nature of digital art practice, and so they were given access to MozEx in order to not only reflect the digital art world itself (with its diverse approach) but to also aiming at including many different audiences who may relate to different kinds/types of work. Diversity of practices meant diversity of audiences.

With the diversity of practices came the diversity of planning. Technical installations were set-up around the festival (at Ravensbourne College in London) and different levels of technical support was needed from the low-key digital screening to more complex installations. The technical challenge was also given by the way we merged MozEx within the Mozilla Festival.

Because the focus of the work was an exploration of the five key issues, the exhibition was spread around the festival venue rather than grouped together in one space. At MozFest there were more than five-hundred activities divided in talks and workshops; these activities were covering many areas of technology and were spread in nine big open-space floors. MozEx connected the festival's thematic elements, visually shaping paths and patterns to its surrounding communities of practitioners. This connectivity between the floors, the topics, the talks and the workshops, was highly reflective of the nature of the festival and its open web community. The curatorial flow underlined the concept of one-web, keeping its multi-facets detailed differences balanced with a unique and positive global connection.

Working in a space that is a non-gallery setting was a challenge and yet, it was an inspiration. We had to be inventive sorting the shape of the exhibit in one day. The MozEx exhibition itself had allocated two spaces dedicated to showing some of the works: fourteen interactive and ten physical pieces. This meant that thirty pieces were set-up throughout the nine floors/levels of the venue, on screens or projections on walls. This shaped the exhibition in a bit of an experience to look\&find; it was a challenge but it actually helped the public in navigating the venue and understanding better the various levels of engagement that the festival was proposing.

\section{ARTISTS CRITICAL ENGAGEMENT}

In this section, we present and collect ten artworks part of the MozEx experience, underlining their diversity in terms of topic, format, inclusion, and public engagement. More work is available to be seen in the digital publication on the Isseu open platform (https://issuu.com/mozfest/docs/mozex). Instead of writing about each piece from our perspective, here we decided to give voice to the artists, allowing a multi-voice exchange also in this paper. So, following are some of the MozEx works, and their related artists-creators' comments and descriptions.

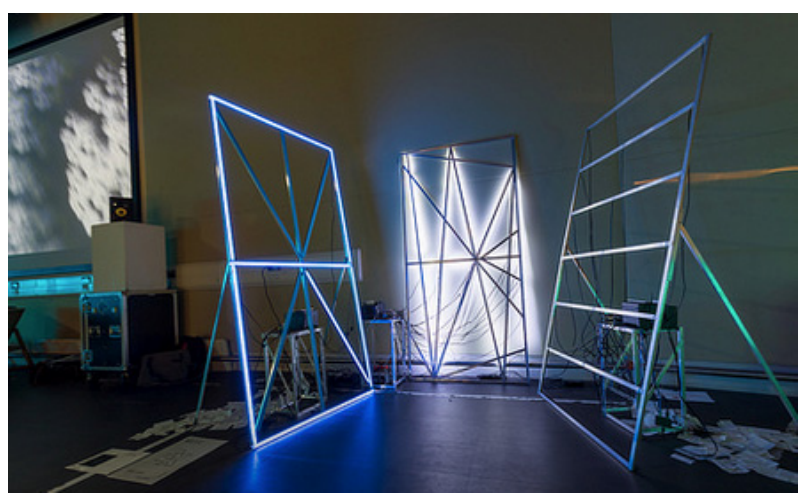

Figure 1: The Treasure Bands - Henrik van Leeuwen

"With the introduction of 5G, at fee mobile data subscriptions and global satellite, weather balloon and drones based internet access the number of access points to the internet seems to be diminishing. Multinationals such as AT\&T, Vodafone, but also Google and Facebook are investing heavily in ways to be the only global (wireless) access point to the internet. Wireless frequencies are becoming the new 'oil' of our connected world. Multinationals are in bidding wars to get the most valuable frequencies. Nation states auction off their frequencies to the highest bidder. These frequencies are milk cows for states. In Treasure Bands we show the hidden world of radio frequencies via an interactive screen-based installation." - Artist: Henrik van Leeuwen

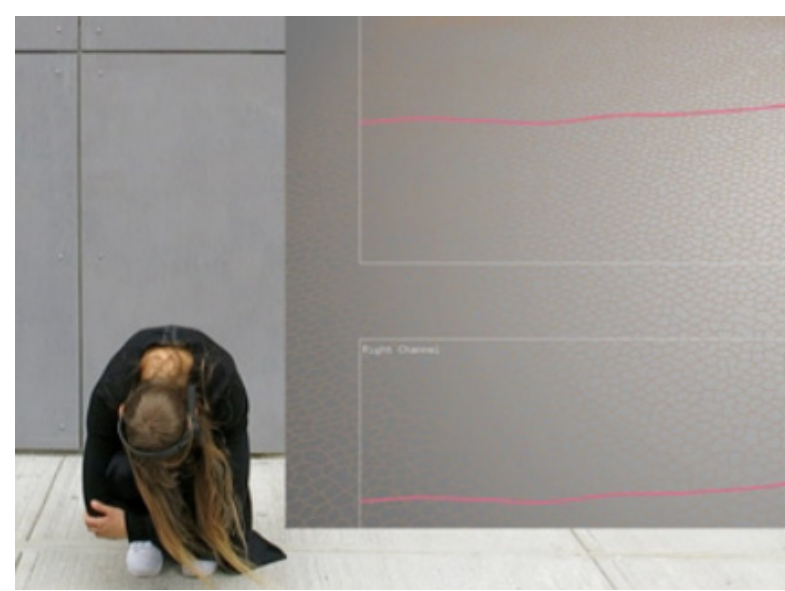

Figure 2: Embodiment of Cognition - Anna Nazo 
"In this piece I experiment with embodiment of cognition. I work with EEG data, creating a metaphorical journey into human's mind and personality. Here I refer to Hayles's posthumanism scenarios on the fate of embodiment in an information age and the cultural and technological construction of the cyborg, which imagines a triumphant transcendence of embodiment and 'privileges informational pattern over material instantiation' (Hayles 1999). I further these ideas translating the recording of electrical activity along the scalp into a sculptural responsive surface, that reacts to a change of a human's brain activity - you perform and transmit your own data. The work uncovers themes of mixed reality and augmentation relating to the body." - Artist: Anna Nazo

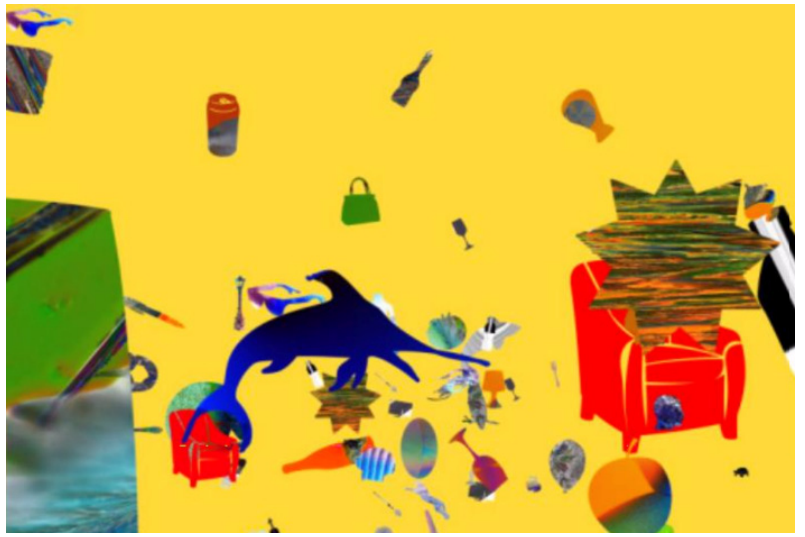

Figure 3: 000 - Alice Dunseath

"OOO is a pair of animated films. I originally created these to be screened as part of Project OOO, a light and video installation inspired by the contemporary philosophical movement known as Object Oriented Ontology by myself and another artist Matteo Mastrandrea. The two films (one yellow and one blue) are designed to be screened opposite each other. Everything in the Blue Im is moving towards the viewer and everything in the yellow Im is moving away, putting the viewer in the middle realm. The objects within the films combine the digital with the organic as each outline is created digitally but each texture inside is microscopic imagery of natural organisms." - Artist: Alice Dunseath

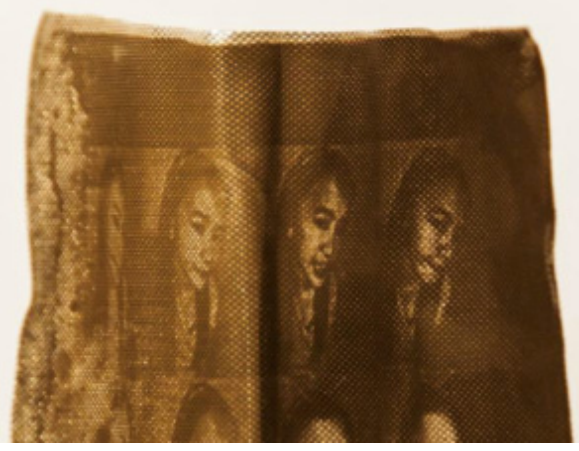

Figure 4: Self-Expanded - Almudena Romero
"The project self-expanded uses real time updates of selfie pictures on Instagram from online feeds. The images are digitally projected onto expanded metal and printed with the nineteenth century technique of wet collodion to inscribe into permanence and uniqueness these ephemeral images. The project reflects on the commodification of the self through technology development and the networked image." - Artist: Almudena Romero

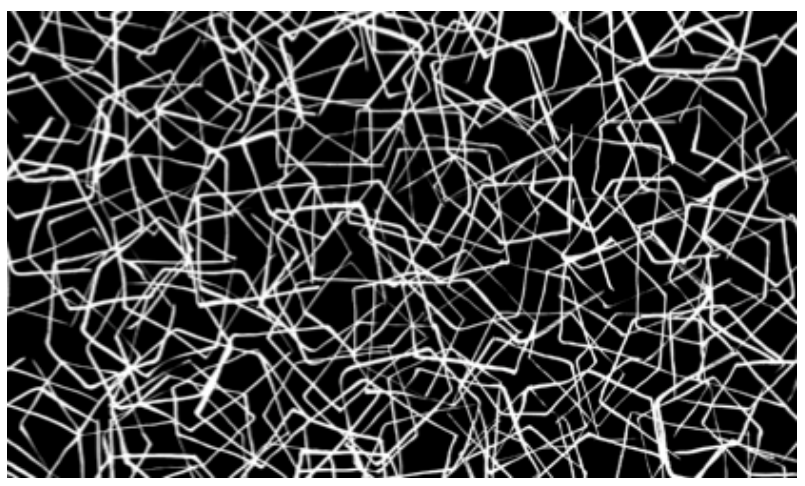

Figure 5: Flown - Esther Rolinson

"This is a series of drawings that relate to the work 'Flown', a programmed light installation developed through drawing. The Flown images show some of the process involved in its conceptual development and practical construction. They range from quickly drawn sketches to detailed works made over a number of months." - Artist: Esther Rolinson

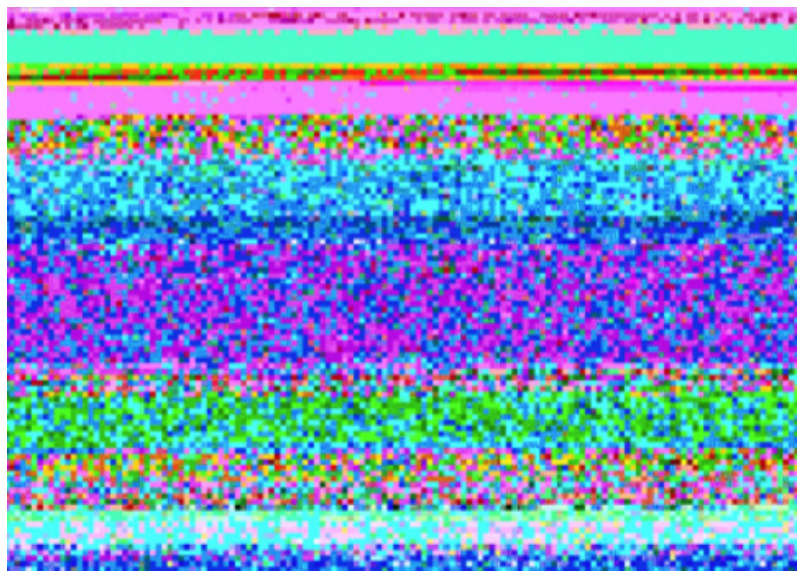

Figure 6: Some of My Favourite Songs: Antonio Roberts

"Some of My Favourite Songs are a series of digital images which examine how data can be interpreted in various unconventional ways. The piece takes various mp3s and uses software written by myself using Pure Data that converts them from an mp3 into a jpg image. The output is very distorted and takes no consideration of factors such as amplitude, song structure, or pitch. Instead the mp3 is treated purely as data" - Artist: Antonio Roberts 


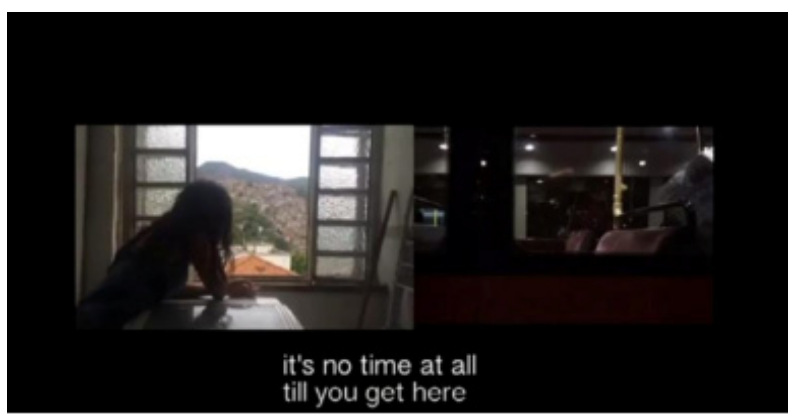

Figure 7: Undersea Cable - Ali Sargent

"A relationship is constructed from two different territories. Undersea Cable is a short Im shot on mobile phones, collecting fragments of Skype conversations from a period of eighteen months between two women; one in London, one in Rio de Janeiro. The audio provides an overlapping centre for the two protagonists' cities, as they describe how they are transforming around them. As the camera journeys through the cities, the two women talk about their lives, families and politics in their different contexts. Images and voices converge in moments of temporary connection and disconnection, suggesting ways in which new technologies create new geographies and forms of intimacy." - Artist: Ali Sargent

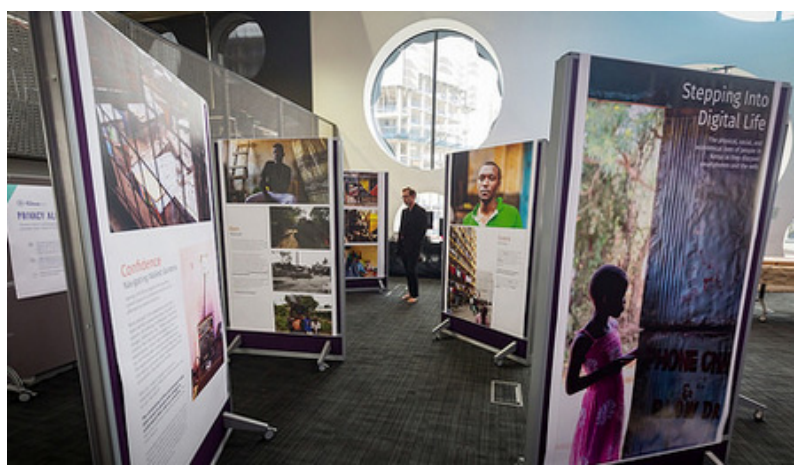

Figure 8: Digital Skills Observatory - Laura de Reynal and Bobby Ritcher

"How are the lives of people affected when we help them come online and understand what's possible? Can research at this level be driven by an open, community-based, capacity-building network?

Witness an exploration through audiovisual experiences of the people, topics, and research behind the Digital Skills Observatory: a year-long, community-driven project following the impact of digital skills training on new smartphone users in Kenya. Become immersed in the physical, social, and economic lives of people in Kenya and how their stories are the basis for experimental methods for learning about smartphones, the internet, and their socioeconomic potential." - Artists: Laura de Reynal and Bobby Ritcher

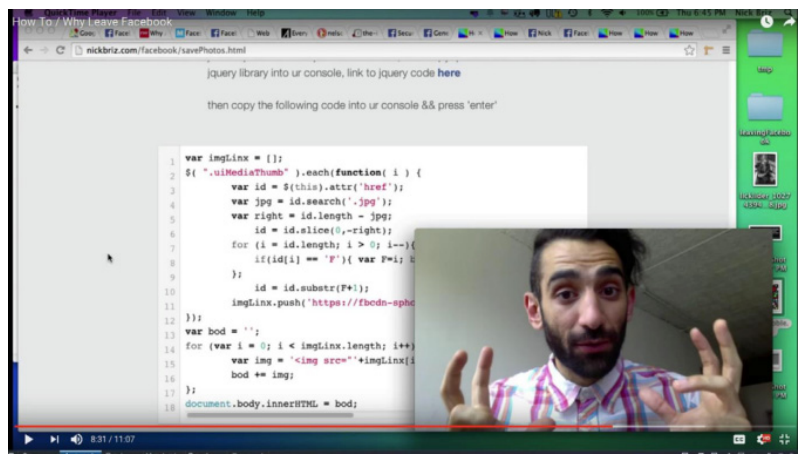

Figure 9: How to / Why to Leave Facebook - Nick Briz

A tutorial/essay video on how-to and why leave Facebook without deleting your account. The tutorial/ essay provides a collection of JavaScript console hacks for downloading data, activity and memories not accessible through the Facebook interface as well as a means for removing all your activity without having to deactivate your account. It's a piece about digital agency and a critique on Facebook's problematic approach to social media." - Artist: Nick Briz

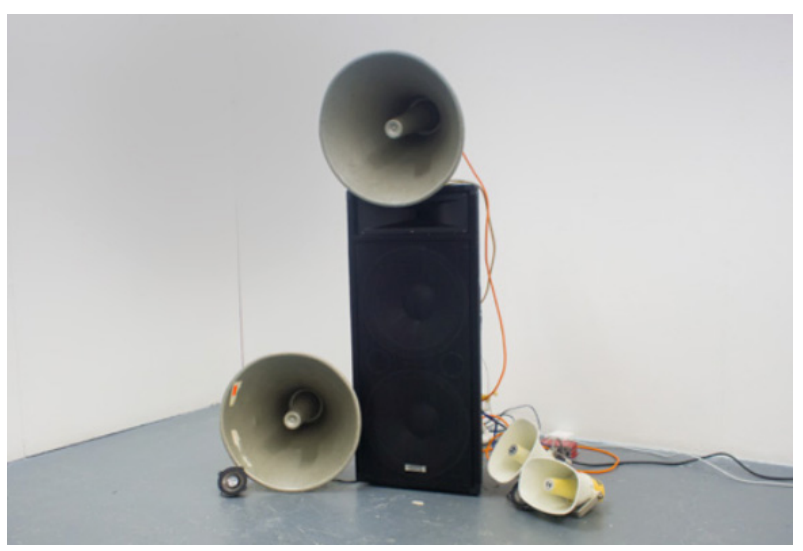

Figure 10: Pardon the Dust - Neale Willis

"When a tweet is deleted, it leaves behind a eighteen digit code, an imprint of a thought or feeling. That number could be considered a sequencer, a data string which when fed through an algorithm becomes a soundscape. Captured from the live twitter stream, the audible output is dictated by what is being removed from the internet at that very moment in time to produce a very public creation of something intended to be obscured, undertaking a process of creation from the act of deletion, a process of revealing through the act of concealment as it algorithmically creates an almost musical score from digital debris. As the machine plays back its composition at a low frequency, the audio can be felt as well as heard, reconfiguring the normally latent digital stream into an unescapable presence." - Artist: Neale Willis 


\section{CONCLUSION}

During the whole process of the project, there was a focus on underlining (and evaluate) the potential of the open internet, especially focusing on the relation that technology and creativity has developed within the web, and how digital art practice is expanding with digital methods and digital spaces.

The exhibit varied in the way it covered the theme and topic. Some artworks had a documentary approach, other artworks were structured as complex systems, others were more technological experiments with a playful vibe, and others were purely using technology to create interesting aesthetics, whilst other developments were only about and towards artificial intelligence and interactive bots.

MozEx engaged the public with the topics of web literacy, digital inclusion and accessibility, privacy, policy and hacking. The diversity or practitioners, from fine artists to coders, from journalists to sound-designers, from human rights organisations to experimental mix-media artists, created a wider frame of visions and voices.

All these different aspects worked together: their differences were their strengths. They created one piece like one web which is not all the same (it keeps its multitude of facets) but it comes together as a whole. Like our society. Perhaps that was the strongest aspect of the installation: the way it reflects the nature of the internet.

In the overall, MozEx was a fundamental curatorial experience to investigate first hand a very open and transparent model of working. Our interest in exploring the use of open tools not only for digital making but also for digital curation and planning, brought us to have different and unexpected (especially for many of the artists) way of working. The use of GitHub, for example, as our main platform for the curatorial practice meant that we were very transparent in the selection process, in the correspondence with the artists, in the exchange between us. All this was accessible to the public in real time, and so it created a very different dynamic from what we (and the public) were accustomed to. This, in a way, can be seen as a further step of freedom of information too, fostering a hub and community for the artists involved, as well as the festival organisers, curators, producers and the public.

As next steps of our digital learning collaboration within Tate, V\&A and Mozilla, we are interested in investigating and reflecting on post-humanism, big data and the Internet of Things (IoT). Looking at the digital arguments for society and community cohesion, we aim to explore the practical application of digital making skills and knowledge to the topics, considering the next generation of practices for designers and artists and how critical voices can be heard and shared for new debates and exchanges.

\section{REFERENCES}

Bearden, S. (2016) Digital Citizenship: A Community Based Approach. Corwin Publisher.

Biggs, S. (2012) Remediating the Social. University of Edinburgh Press.

Cook, S. (2016) Documents of Contemporary Art. MIT Press.

Graham, B. and Cook, S. (2010) Rethinking Curating. Art After New Media. MIT Press.

Greengard, S. (2015) The Internet of Things. The MIT Press.

Hayles, K. (1999) How We Became Posthuman: Virtual Bodies in Cybernetics, Literature, and Informatics. University of Chicago Press.

Krysa, J. (2006) Curating Immateriality. Autonomedia.

Mihailidis, P. (2014) Media Literacy and the Emerging Citizen: Youth, Engagement and Participation in Digital Culture. Peter Lang International Academic Publishers.

Najar, P. (2003) Posthumanism. Polity.

Quaranta, D (2013) Beyond New Media Art. LINK Editions.

Schuber, K. and McClean, D. (2002) Dear Images: Art, Copyright and Culture. Ridinghouse.

Soep, E. (2014) Participatory Politics: NextGeneration Tactics to Remake Public Spheres (The John D. and Catherine T. MacArthur Foundation Reports on Digital Media and Learning). The MIT Press.

Stackowiak, R, Licth, A., Mantha, V., and Nagode, L. (2015) Big Data and The Internet of Things: Enterprise Information Architecture for A New Age. Apress.

\section{Online Ressources}

Creative Commons: https://creativecommons.org

GitHub: https://github.com

MozEx, Issuu:

https://issuu.com/mozfest/docs/mozex 